Research Paper

\title{
The Effects of Perioperative Anesthesia and Analgesia on Immune Function in Patients Undergoing Breast Cancer Resection: A Prospective Randomized Study
}

Jin Sun Cho ${ }^{1 *}$, Mi-Hyang Lee2,3*, Seung Il Kim, Seho Park ${ }^{4}$, Hyung Seok Park ${ }^{4}$, Ein Oh¹, Jong Ho Lee², 5, 6®, and Bon-Nyeo Koo ${ }^{1 凶}$

1. Department of Anesthesiology and Pain Medicine, Anesthesia and Pain Research Institute, Yonsei University College of Medicine, Seoul, Republic of Korea;

2. National Leading Research Laboratory of Clinical Nutrigenetics/Nutrigenomics, Department of Food and Nutrition, College of Human Ecology, Yonsei University, Seoul, Republic of Korea;

3. Korea Ginseng Corporation Research Institute, Korea Ginseng Corporation, Daejeon, Republic of Korea;

4. Department of Surgery, Yonsei University College of Medicine, Seoul, Republic of Korea;

5. Department of Food and Nutrition, Brain Korea 21 PLUS Project, College of Human Ecology, Yonsei University, Seoul, Republic of Korea;

6. Research Center for Silver Science, Institute of Symbiotic Life-TECH, Yonsei University, Seoul, Republic of Korea.

* Jin Sun Cho and Mi-Hyang Lee are co-first authors.

$\triangle$ Corresponding authors: Jong Ho Lee, Department of Food and Nutrition, Yonsei University, 50 Yonsei-ro, Seodaemun-gu, Seoul 03722, Republic of Korea Fax: +82-2-364-2951/ Tel: +82-2-2123-8385/ E-mail: jhleeb@younsei.ac.kr Bon-Nyeo Koo, Department of Anesthesiology and Pain Medicine, Anesthesia and Pain Research Institute, Yonsei University College of Medicine, 50-1 Yonsei-ro, Seodaemun-gu, Seoul 03722, Republic of Korea Fax: +82-2-364-2951/ Tel: +82-2-2227-3835/ E-mail: koobn@yuhs.ac

(C) Ivyspring International Publisher. This is an open access article distributed under the terms of the Creative Commons Attribution (CC BY-NC) license (https://creativecommons.org/licenses/by-nc/4.0/). See http://ivyspring.com/terms for full terms and conditions.

Received: 2017.03.14; Accepted: 2017.06.18; Published: 2017.08.18

\begin{abstract}
Introduction: Perioperative anesthesia and analgesia exacerbate immunosuppression in immunocompromised cancer patients. The natural killer (NK) cell is a critical part of anti-tumor immunity. We compared the effects of two different anesthesia and analgesia methods on the NK cell cytotoxicity (NKCC) in patients undergoing breast cancer surgery.

Methods: Fifty patients undergoing breast cancer resection were randomly assigned to receive propofol-remifentanil anesthesia with postoperative ketorolac analgesia (Propofol-ketorolac groups) or sevoflurane-remifentanil anesthesia with postoperative fentanyl analgesia (Sevoflurane-fentanyl group). The primary outcome was NKCC, which was measured before and $24 \mathrm{~h}$ after surgery. Post-surgical pain scores and inflammatory responses measured by white blood cell, neutrophil, and lymphocyte counts were assessed. Cancer recurrence or metastasis was evaluated with ultrasound and whole body bone scan every 6 months for 2 years after surgery.

Results: The baseline NKCC (\%) was comparable between the two groups $(P=0.082)$. Compared with the baseline value, NKCC (\%) increased in the Propofol-ketorolac group [15.2 (3.2) to 20.1 (3.5), $P=$ 0.048], whereas it decreased in the Sevoflurane-fentanyl group [19.5 (2.8) to $16.4(1.9), P=0.032]$. The change of NKCC over time was significantly different between the groups $(P=0.048)$. Pain scores during $48 \mathrm{~h}$ after surgery and post-surgical inflammatory responses were comparable between the groups. One patient in the Sevoflurane-fentanyl group had recurrence in the contralateral breast and no metastasis was found in either group.

Conclusions: Propofol anesthesia with postoperative ketorolac analgesia demonstrated a favorable impact on immune function by preserving NKCC compared with sevoflurane anesthesia and postoperative fentanyl analgesia in patients undergoing breast cancer surgery.
\end{abstract}

Key words: anesthesia; analgesia; breast cancer; immunity; natural killer cell.

\section{Introduction}

Surgical resection is one of the primary treatments for solid tumors, but the dissemination of tumor cells into the blood and lymphatic systems inevitably occurs during surgery. Whether the 
residual tumor cells lead to clinical deterioration depends on the balance between perioperative factors promoting cancer survival and growth and the host's anti-tumor defenses [1]. Surgery-related neuroendocrine stress responses, anesthetics, and opioid analgesics are factors known to adversely affect the anti-tumor immune defenses [2].

Natural killer (NK) cells are a critical part of innate immunity, acting as the main defense against the spread of cancer [3]. Reduced NK cell cytotoxicity (NKCC) is associated with poor cancer prognosis in breast, colon, and prostate cancers [4-6]. Especially in breast cancer patients, the level of NKCC is inversely correlated with the stage and metastasis of cancer [4].

To date, there have been few prospective studies comparing the effects of perioperative anesthetic and analgesic agents on NKCC in patients undergoing cancer surgery. Previous experimental studies have shown both detrimental and protective effects of certain anesthetics and analgesics on immune function. Volatile anesthetic agents, including sevoflurane and desflurane, decreased NKCC [7], whereas propofol did not suppress NKCC [8]. Fentanyl suppressed NK cell function [9], whereas non-steroidal anti-inflammatory drugs (NASIDs) reversed NKCC suppression [10]. Based on these results, we hypothesized that avoiding volatile anesthetics and opioid analgesics might attenuate the immunosuppression in the perioperative periods. In this prospective randomized study, we compared the effects of two different anesthetic and analgesic methods on NKCC in patients undergoing breast cancer surgery.

\section{Patients and Methods}

This study was approved by the Institutional Review Board and Hospital Research Ethics Committee of Severance Hospital, Yonsei University Health System, Seoul, Korea, on February 2014 (\#4-2013-0937). It was registered at clinicaltrial.gov on March 2014 (NCT02089178). Patients (20-65 years old) who underwent elective surgery for breast cancer and had an American Society of Anesthesiologists (ASA) physical status classification of I to III were included. The exclusion criteria were renal or hepatic impairment, a body mass index $>35 \mathrm{~kg} / \mathrm{m}^{2}$, immunosuppressive therapy, immune disorders, steroid administration within the last six months, metastasis, or radiotherapy or chemotherapy before surgery. Written formed consent was obtained from all of patients. A total of 50 patients were randomly assigned into one of the study groups ( 25 patients each) using a computer-generated random number table. In the Propofol-ketorolac group, patients were anesthetized with propofol and remifentanil and received ketorolac after surgery. In the Sevo-fentanyl group, patients were anesthetized with sevoflurane and remifentanil and received fentanyl postoperatively. Assignments were concealed in sealed envelopes and the randomization was not stratified or blocked.

In the operating theatre, patients received glycopyrrolate $0.2 \mathrm{mg}$ and were applied with routine ASA monitoring, including electrocardiography, peripheral oxygen saturation, and blood pressure. Before anesthetic induction, a peripheral venous cannula was inserted to obtain a blood sample before and after surgery. In the Propofol-ketorolac group, propofol was administered using a target-controlled infusion (Orchestra ${ }^{\circledR}$ Base Primea, Fresenius Vial, Sévres, France) with the modified Marsh model. A target plasma concentration of propofol was $4 \mu \mathrm{g} / \mathrm{ml}$ initially, and then adjusted in $0.2 \mu \mathrm{g} / \mathrm{ml}$ increments. In the Sevo-fentanyl group, anesthesia was induced with propofol 1.5 to $2 \mu \mathrm{g} / \mathrm{kg}$ and maintained with sevoflurane. The concentrations of propofol and sevoflurane were titrated to maintain the bispectral index score in the range of 40-60. In addition to anesthetic agents, remifentanil was infused in both groups to maintain hemodynamic stability intraoperatively. Low-dose remifentanil infusion did not impair NKCC [11]. Remifentanil was infused using a target-controlled infusion to achieve an effect-site concentration of $1.5 \mathrm{ng} / \mathrm{ml}$ at induction and then between 3 and $5 \mathrm{ng} / \mathrm{ml}$ for maintenance. Rocuronium $0.6 \mathrm{mg} / \mathrm{kg}$ was administered to facilitate tracheal intubation. Mean blood pressure and heart rate were maintained within $25 \%$ of baseline. At the end of the surgery, all patients received neostigmine $40 \mu \mathrm{g} / \mathrm{kg}$ and glycopyrrolate $5 \mu \mathrm{g} / \mathrm{kg}$ for reversal of neuromuscular block and ramosetron $0.3 \mathrm{mg}$ for postoperative nausea and vomiting prophylaxis. At the end of surgery, the Propofol-ketorolac group received ketorolac $60 \mathrm{mg}$ and the Sevo-fentanyl group received fentanyl $50 \mu \mathrm{g}$ for acute pain relief.

The primary aim was to compare the effects of two anesthetic and analgesic methods on the immune function assessed by NKCC, measured preoperatively and at $24 \mathrm{~h}$ postoperatively. Other outcome measures included postoperative pain scores, IL-2 levels, and inflammatory responses assessed by white blood cell, neutrophil, and lymphocyte counts. The incidence of cancer recurrence or metastasis was evaluated with a breast ultrasound, abdomen ultrasound, and whole body bone scan every 6 months after surgery.

\section{Assay for natural killer cell cytotoxicity}

Blood samples were obtained before and at $24 \mathrm{~h}$ after surgery. Whole blood was mixed with the same volume of RPMI 1640 (Gibco, Invitrogen Co, USA), 
and the mixture was laid on Histipaque ${ }^{\circledR}-1077$ (Sigma, CA, USA) and centrifuged (2000 rpm for $20 \mathrm{~min}$ at $10^{\circ} \mathrm{C}$ ). Then, a thin layer of peripheral blood mononuclear cells (PBMCs) was harvested, washed twice with RPMI 1640, and resuspended in RPMI 1640 containing streptomycin. NKCC was assessed using the CytoTox $96^{\circledR}$ Non-Radioactive Cytotoxicity Assay Kit (Promega Co., WI, USA). This colorimetric assay quantitatively measures lactate dehydrogenase (LDH), a stable cytosolic enzyme that is released upon cell lysis, in much the same way that ${ }^{51} \mathrm{Cr}$ is released in a radioactive assay. PBMCs (effector cell) and K562 cells (targeted cell; $2 \times 10^{4}$ cells/well) were seeded in the well in a ratio of 1.25:1 and incubated overnight at $37^{\circ} \mathrm{C}$ in $5 \% \mathrm{CO}_{2}$. Finally, the NKCC of effector cells was measured with a 2030 multilabel reader $\left(\right.$ Victor $^{\mathrm{TM}}$ X5, PerkinElmer, MA, USA) at $490 \mathrm{~nm}$, and the cytotoxicity percentage was calculated with as [12]:

$$
\begin{gathered}
\% \text { Cytotoxicity }=[(\text { Experimental }- \text { Effector } \\
\text { Spontaneous - Target Spontaneous) / (Target } \\
\text { Maximum - Target Spontaneous) }] \times 100
\end{gathered}
$$

The maximum LDH release from target cells was measured by using the lysis solution (Triton ${ }^{\circledR} \mathrm{X}-100$ ), which should yield complete lysis of target cells and subsequent release of cytoplasmic LDH into the surrounding culture medium.

\section{Assay for interleukin-2}

IL-2 was measured in serum using a commercial ELISA kit (Quantikine Human IL-2 ELISA Kit; R\&D System Inc., MN, USA) preoperatively and at $24 \mathrm{~h}$ after surgery. The absorbance was read at $450 \mathrm{~nm}$ using a Spectra Max 190 micro-plate reader (Molecular Devices, CA, USA).

\section{Pain scores}

Pain scores were assessed using a 11-point numerical rating scale (NRS, $0=$ no pain to $10=$ worst pain) at postoperative $30 \mathrm{~min}, 6 \mathrm{~h}, 24 \mathrm{~h}$, and $48 \mathrm{~h}$. For immediate postoperative analgesia, the Propofol-ketorolac group received ketorolac $60 \mathrm{mg}$ and the Sevo-fentanyl group received fentanyl $50 \mu \mathrm{g}$ at the end of surgery. In the post-anesthesia care unit, propacetamol $2 \mathrm{~g}$ in the Propofol-ketorolac group or fentanyl $50 \mu \mathrm{g}$ in the Sevo-fentanyl group was available as an additional analgesic for patients with a NRS $\geq 4$. In the ward, both groups received tramadol $50 \mathrm{mg}$ as a rescue analgesic, which does not suppress NKCC [13].

\section{Statistics}

No previous study was available to inform assumptions regarding changes of NKCC following two different anesthetic and analgesic methods. We calculated a sample size based on preliminary results for the first five patients of each group, and estimated that 22 patients in each group would be required to detect a mean difference of $10 \%$ and standard deviation of $10 \%$ in the NKCC after surgery with $90 \%$ power at a significance of $P<0.05$. We factored in a $10 \%$ dropout rate and enrolled 25 patients in each group.

Statistical analyses were performed with IBM SPSS 20.0 (IBM Corp., Armonk, NY, USA) and SAS 9.2 (SAS Institute Inc., Cary, NC, USA). Continuous variables were analyzed with the independent $t$-test or Mann-Whitney U test, after testing for normality of distribution using Kolmogorov-Smirnov test. Categorical variables were analyzed with $\chi^{2}$ test or Fisher exact test. Variables measured repeatedly, such as NKCC, IL-2, total leukocyte, neutrophil, and lymphocyte counts, and neutrophil-lymphocyte-ratio (NLR) were analyzed with a linear mixed model, with patient indicator as a random effect and with group, time, and group-by-time as fixed effects. The group-by-time interaction assesses whether the change over time differs between groups. Post-hoc analyses with the Bonferroni correction were performed for multiple comparisons when variables with repeated measures showed significant differences between groups. A P value $<0.05$ was considered statistically significant.

\section{Results}

Of 50 patients enrolled, one patient in each group was eliminated due to concurrent breast reconstruction surgery. The remaining 48 patients completed the study without any complications. Patient characteristics and operation details were comparable between the two groups (Table 1).

\section{Natural killer cell cytotoxicity}

The baseline NKCC (\%) was not statistically different between the two groups $(P=0.082)$. Compared to the baseline, NKCC (\%) increased after surgery in the Propofol-ketorolac group [mean (SEM) preoperative to postoperative; 15.2 (3.2) to 20.1 (3.5), $P$ $=0.048]$, whereas it decreased in the Sevo-fentanyl group [19.5 (2.8) to 16.4 (1.9), $P=0.032$ ]. The change of NKCC over time was significantly different between the groups $(P=0.048)$ (Figure 1$)$.

\section{Serum concentration of interleukin-2}

No significant postoperative change was seen in IL-2 levels in either group [median (IQR) preoperative to postoperative; $2.75(1.61,4.97)$ to $3.16(1.97,5.52), P$ $=0.721$ in the Propofol- ketorolac group, and 2.65 $(2.15,3.96)$ to $2.81(2.00,4.62), P=0.523$ in the Sevofentanyl group]. The change of IL-2 levels over time was not significant between the groups $(P=0.620)$. 
Table 1. Patient characteristics and operation details

\begin{tabular}{lll}
\hline Variables & $\begin{array}{l}\text { Propofol-ketorolac group } \\
(\mathrm{n}=24)\end{array}$ & $\begin{array}{l}\text { Sevo-fentanyl group } \\
(\mathrm{n}=24)\end{array}$ \\
\hline Age $($ years $)$ & $55.4 \pm 7.0$ & $55.7 \pm 12.9$ \\
BMI $\left(\mathrm{kg} / \mathrm{m}^{2}\right)$ & $23.6 \pm 3.0$ & $24.3 \pm 3.7$ \\
ASA class II/III & $9 / 0$ & $6 / 3$ \\
Type of surgery & & 0.923 \\
Partial mastectomy & 7 & 0.500 \\
Total mastectomy & 14 & 0.165 \\
Radical mastectomy & 3 & 0.360 \\
Lymph node involvement & & 0.233 \\
$\quad$ Positive/ negative & $13 / 11$ & 0.562 \\
Duration of operation $(\mathrm{min})$ & $101.6 \pm 30.9$ & 0.296 \\
Duration of anesthesia $(\mathrm{min})$ & $132.8 \pm 28.2$ & $99.7 \pm 33.0$ \\
Dose of remifentanil $(\mu \mathrm{g} / \mathrm{kg} / \mathrm{min})$ & $0.09 \pm 0.02$ & $127.0 \pm 34.7$ \\
\hline
\end{tabular}

Values are mean \pm standard deviation or number.

Propofol-ketorolac group, propofol-remifentanil anesthesia and postoperative ketorolac analgesia group; Sevo-fentanyl group, sevoflurane-remifentanil anesthesia and postoperative fentanyl analgesia group; BMI, body mass index; ASA class, American Society of Anesthesiologists physical status classification.
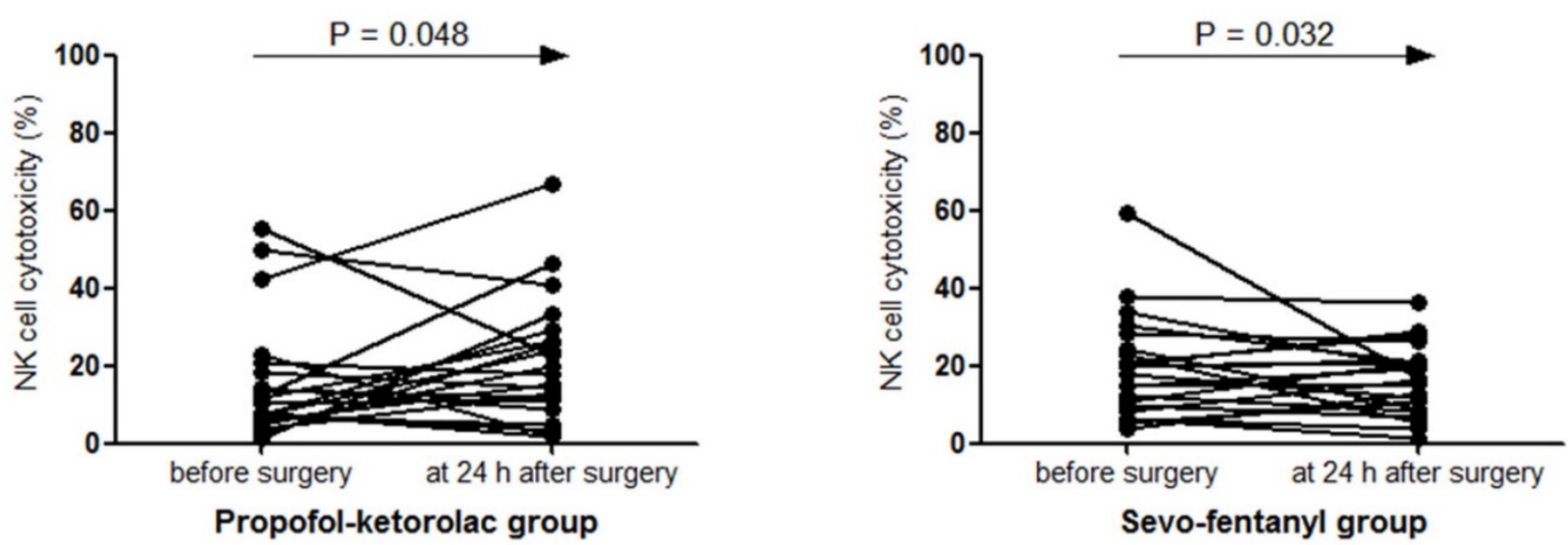

Figure 1. Natural killer cell cytotoxicity before and after surgery NK cell, natural killer cell; Propofol-ketorolac group, propofol-remifentanil anesthesia and postoperative ketorolac analgesia group; Sevo-fentanyl group, sevoflurane-remifentanil anesthesia and postoperative fentanyl analgesia group. The change of NK cell cytotoxicity over time was significantly different between the groups ( $\mathrm{P}$ Group $\times$ Time $=0.048)$.

\section{Inflammatory response}

The changes of the total leukocyte, neutrophil, and lymphocyte counts and NLR over time were not significant between the groups (Table 2). Lymphocyte counts after surgery decreased in both groups compared to the baseline, but the difference was significant only in the Sevo-fentanyl group $(P=0.037)$.

\section{Pain score}

Pain scores during the postoperative $48 \mathrm{~h}$ were comparable between the two groups. In the post-anesthesia care unit, 8 patients in the Propofol-ketorolac group received propacetamol and 12 patients in the Sevo-fentanyl group received fentanyl as an additional analgesic. The number of patients requiring tramadol in the ward and total doses given were comparable between two groups (Table 3).

\section{Postoperative outcomes}

One patient in the Sevo-fentanyl group had recurrence in the contralateral breast 18 months after surgery, and underwent a partial mastectomy. No patient had metastasis in either group within two years after surgery.

\section{Discussion}

During breast cancer surgery, patients who received propofol-remifentanil anesthesia and postoperative ketorolac analgesia exhibited preserved NKCC compared with those who received sevoflurane-remifentanil anesthesia and postoperative fentanyl analgesia. Postoperative inflammatory responses and the incidences of short-term cancer recurrence and metastasis were not different between the two anesthetic and analgesic methods. 
Table 2. Total leukocyte, neutrophil, and lymphocyte counts

\begin{tabular}{llll}
\hline Variables & Time points & Propofol-ketorolac group $(\mathrm{n}=24)$ & Sevo-fentanyl group $(\mathrm{n}=24)$ \\
\hline Leukocyte & before surgery & $6.05 \pm 1.47$ & $6.83 \pm 1.43$ \\
$\left(10^{-3} / \mu \mathrm{L}\right)$ & at 24 h after surgery & $7.78 \pm 1.62^{*}$ & $8.57 \pm 1.88^{*}$ \\
Neutrophil & before surgery & $3.37 \pm 1.27$ & $4.09 \pm 1.07$ \\
$\left(10^{-3} / \mu \mathrm{L}\right)$ & at 24 h after surgery & $5.41 \pm 1.35^{*}$ & $6.13 \pm 1.56^{*}$ \\
Lymphocyte & before surgery & $1.99 \pm 0.51$ & $2.15 \pm 0.71$ \\
$\left(10^{-3} / \mu \mathrm{L}\right)$ & at 24 h after surgery & $1.74 \pm 0.51$ & $1.75 \pm 0.66^{*}$ \\
Neutrophil to lymphocyte ratio & before surgery & $1.76 \pm 0.69$ & 0.988 \\
& at 24 h after surgery & $3.37 \pm 1.27^{*}$ & 0.538 \\
\end{tabular}

Values are mean \pm standard deviation.

Propofol-ketorolac group, propofol-remifentanil anesthesia and postoperative ketorolac analgesia group; Sevo-fentanyl group, sevoflurane-remifentanil anesthesia and postoperative fentanyl analgesia group; $\mathrm{P}_{\text {Group } \times \text { Time, }} \mathrm{P}$-value for the group $\times$ time interaction in the linear mixed model.

The baseline values were not different between the two groups. ${ }^{*} P<0.05$ vs. before surgery.

Table 3. Pain scores and additional analgesic requirements

\begin{tabular}{|c|c|c|c|}
\hline & Propofol-ketorolac group $(\mathrm{n}=24)$ & Sevo-fentanyl group $(n=24)$ & $P$ value \\
\hline \multicolumn{4}{|l|}{ Pain score } \\
\hline at post-op $30 \mathrm{~min}$ & $2.8 \pm 0.8$ & $2.7 \pm 0.6$ & 0.476 \\
\hline at post-op $6 \mathrm{~h}$ & $2.2 \pm 0.7$ & $2.3 \pm 1.1$ & 0.642 \\
\hline at post-op $24 \mathrm{~h}$ & $1.9 \pm 0.6$ & $2.1 \pm 0.7$ & 0.315 \\
\hline at post-op $48 \mathrm{~h}$ & $1.6 \pm 0.7$ & $1.6 \pm 0.8$ & 0.941 \\
\hline \multicolumn{4}{|l|}{ Worst pain score } \\
\hline during post-op $30 \mathrm{~min}$ & $4.0 \pm 1.5$ & $4.0 \pm 1.4$ & 0.923 \\
\hline during post-op $30 \mathrm{~min}-6 \mathrm{~h}$ & $4.3 \pm 1.9$ & $5.0 \pm 2.5$ & 0.271 \\
\hline during post-op 6-24 h & $2.5 \pm 1.3$ & $3.3 \pm 1.7$ & 0.104 \\
\hline during post-op $24-48 \mathrm{~h}$ & $1.9 \pm 0.4$ & $2.0 \pm 1.0$ & 0.828 \\
\hline \multicolumn{4}{|l|}{ Analgesic drugs } \\
\hline at the end of surgery (ketorolac, mg) (n)* & $60(24)$ & 0 & $>0.999$ \\
\hline$($ fentanyl, $\mu \mathrm{g})(\mathrm{n})^{*}$ & 0 & $50(24)$ & $>0.999$ \\
\hline during post-op $30 \mathrm{~min}$ (propacetamol, g) (n) ${ }^{*}$ & $2(8)$ & 0 & $>0.999$ \\
\hline$($ fentanyl, $\mu \mathrm{g})(\mathrm{n})^{*}$ & 0 & $75.0 \pm 39.9(12)$ & $>0.999$ \\
\hline during post-op $30 \mathrm{~min}-6 \mathrm{~h}$ (tramadol, $\mathrm{mg})(\mathrm{n})^{*}$ & $47.9 \pm 7.2(12)$ & $53.8 \pm 13.9(13)$ & $0.199(0.773)$ \\
\hline during post-op 6-24 h (tramadol, mg) (n)* & $45.0 \pm 11.2(5)$ & $60.0 \pm 22.4(5)$ & $0.217(>0.999)$ \\
\hline during post-op $24-48 \mathrm{~h}$ (tramadol, mg) (n) * & 0 & $50(1)$ & $0.35(0.75)$ \\
\hline
\end{tabular}

Values are mean \pm standard deviation or number.

Propofol-ketorolac group, propofol-remifentanil anesthesia and postoperative ketorolac analgesia group; Sevo-fentanyl group, sevoflurane-remifentanil anesthesia and postoperative fentanyl analgesia group; post-op, postoperative; Pain score, a numerical pain intensity scale $\left(0=\right.$ no pain, $10=$ the worst pain); $(\mathrm{n})^{*}$, number of patients requiring analgesic drugs.

Compelling clinical and experimental evidences have suggested that surgery and anesthesia cause a transient period of immunosuppression, which may encourage both the implantation of surgically disseminated neoplastic cells and the growth of existing micro-metastases [1, 2]. Anesthesia can interact with the immune system, facilitating or hindering tumor growth and metastasis. NK cells, $\mathrm{CD}^{-} \mathrm{CD}^{-} 6^{+}$lymphocytes, have direct cellular cytotoxicity against tumor cells and act as the body's main defense against local tumor growth and metastasis.[1,3] Patients diagnosed with breast cancer have inhibition and significantly lower levels of NKCC than do healthy patients. Furthermore, patients with more advanced breast cancer (stage 4) have an increased proportion of immature and non-cytotoxic NK cells in the blood than those with stage 1-3 [4].

Anesthetic agents have exhibited different effects on NKCC in animal and human studies. Propofol preserved NKCC and cytotoxic T lymphocyte activity and suppressed tumor growth. [8, 14] Propofol has cyclooxygenase (COX)-2 inhibiting activity, reducing the production of prostaglandin $E_{2}$ $\left(\mathrm{PGE}_{2}\right)$, a mediator of pain and inflammation that inhibits NKCC [15]. In contrast that propofol has only minor effects on NK cells at clinically relevant concentrations, volatile anesthetics have the dose- and time-dependent suppressive effects on NK cells and T lymphocytes. Isoflurane [16], sevoflurane [7], and desflurane [7] were demonstrated to suppress NKCC. In a rat model of lung tumor, halothane reduced NKCC and increased lung tumor retention and metastases, whereas propofol preserved NKCC [8]. These attributes of propofol and sevoflurane are consistent with our results, which showed increased NKCC in the Propofol-ketorolac group and decreased NKCC in the Sevo-fentanyl group.

Both pain and opioid analgeiscs are known to cause immunosuppression [1, 2]. Fentanyl and morphine depressed NK cell function and markedly increased tumor burden, whereas ketorolac reversed 
NKCC suppression [9, 10]. NSAIDs inhibit prostaglandin synthesis via inhibition of the COX enzyme and COX-2 inhibitors have anti-tumor and anti-angiogenic properties [17]. As our two groups showed comparable analgesic efficacies, the impact of pain on immune function should have been the same in both groups, eliminating pain as a contributing factor to different NKCC between the groups. Therefore, immunosuppressive effect of fentanyl might contribute to decreased NKCC in the Sevo-fentanyl group compared with preseved NKCC in the Propofol-ketorolac group.

Antitumor responses in NKCC are activated by various cytokines; and among those, IL-2 is an important activator of NK cells [18]. IL-2 administration reversed the NKCC suppression associated with surgery and significantly decreased tumor incidence in an animal model [10]. In addition, IL-2 activated human NK cells, effectively killing colon carcinoma, in vitro [19]. We assessed IL-2 level to exclude the possibility of different IL-2 level between the groups before surgery and investigate if NKCC change after surgery would be associated with IL-2 change. We observed the significant increase of IL-2 in both groups after surgery compared to before surgery, but no significant difference was found between the two groups.

Anesthetic and analgesic agents may affect the concentrations of immunocompetent cells, resulting in lymphopenia and neutrophilia [20]. A high NLR is considered as a prognostic indicator for breast cancer [21]. Suppression of lymphocytes is proportional to the dose and duration of anesthetics use. Volatile anesthetics increased the neutrophil and decreased the lymphocyte [7, 22], and propofol also decreased peripheral lymphocytes counts by reducing proliferative responses of lymphocytes [23]. However, propofol provided more effective protection for circulating lymphocytes than sevoflurane [24]. Fentanyl induced a time-dependent apoptosis of lymphocytes [25], and flurbiprofen showed similar effects on lymphocyte level compared with fentanyl [26]. In the present study, the neutrophil count and NLR increased significantly after surgery in both groups, whereas the lymphocyte decreased significantly only in the Sevo-fentanyl group. Our findings suggest that sevoflurane and fentanyl induced more suppressive effect on lymphocytes compared to propofol and ketorolac.

As detrimental effects of volatile anesthesia and opioids on immune function have been identified, regional anesthesia has been tried in an attempt to reduce volatile anesthetics or opioid use. In breast cancer surgery, patients who received propofol and paravertebral block showed preserved NKCC compared to those who received sevoflurane and opioids [27]. However, the addition of spinal blockade to halothane did not attenuate NKCC suppression compared to halothane anesthesia alone or combined with systemic morphine [28]. A recent meta-analysis showed no association between regional anesthesia and NK cell function [29]. Whereas there are contraindications such as coagulopathy or inflammation and the possibility of failure to perform regional anesthesia, intravenous or volatile anesthesia can be performed with little difficulties and few contraindications. Considering the effects on immune function, the anesthetic agent of choice in cancer surgery would be propofol rather than volatile anesthetics. In addition, non-opioid analgesics may be ideal for the restoration of perioperative immune competence rather than opioid.

Our study has some limitations. First, due to the study design, the operating room staff could not be blinded to the group allocation. However, the investigators who conducted follow-ups postoperatively, including analysis of laboratory findings and assessments of pain intensity, were completely unaware of the patient's group assignment. Second, we used remifentanil for intraoperative hemodynamic stability and tramadol for postoperative pain control in both groups. Although remifentanil and tramadol did not impair NKCC $[11,13]$ and the doses were comparable between the groups in the present study, the possible impacts of remifentanil and tramadol on NKCC cannot be excluded. Third, we could not discriminate the respective effects of each drug on NKCC and inflammatory responses. The differences of postoperative NKCC between the groups might be attributed to the combined effects of propofol-ketorolac and sevoflurane-fentanyl. Forth, we used PBMCs as effector cells instead of isolating NK cells. Although NK cell proportion varies individually, the cytotoxic activity of PBMC and separated NK cell samples was reported to be correlated, indicating that both PBMC and separated NK cell measurement methods are equally effective tools for investigation of NK cell activity [30]. NKCC using PBMC can provide natural circumstance like in vivo environment [31]. Last, although cancer metastasis within two year after surgery did not occur in the present study, further evaluation of long-term outcomes are needed to make a conclusion about cancer recurrence or metastasis.

In conclusion, propofol anesthesia and postoperative ketorolac analgesia in breast cancer surgery demonstrated a better effect on the immune function by preserving NKCC compared to sevoflurane anesthesia and postoperative fentanyl 
analgesia. The findings of the present study are consistent with the hypothesis that avoiding volatile anesthetics and opioids could reduce the immunosuppression during surgery. Careful selection of anesthetic and analgesic agents may influence immune functions and postoperative outcomes. Further studies to find anesthetic and analgesic methods which mitigate immunesuppression in cancer surgery are warranted.

\section{Acknowledgements}

Financial support and sponsorship: This work was supported by the National Research Foundation of Korea (NRF) grant funded by the Korea government (MSIP) (No. 2014R1A2A2A01007289).

Trial registration: Clinicaltrials.gov identifier: NCT02089178.

\section{Competing Interests}

The authors have declared that no competing interest exists.

\section{References}

1 Snyder GL, Greenberg S. Effect of anaesthetic technique and other perioperative factors on cancer recurrence. Br J Anaesth. 2010; 105:106-115.

2 Kurosawa S. Anesthesia in patients with cancer disorders. Curr Opin Anaesthesiol. 2012; 25:376-384.

3 Chester C, Fritsch K, Kohrt HE. Natural Killer Cell Immunomodulation: Targeting Activating, Inhibitory, and Co-stimulatory Receptor Signaling for Cancer Immunotherapy. Front Immunol. 2015; 6:601.

4 Konjevic G, Spuzic I. Stage dependence of NK cell activity and its modulation by interleukin 2 in patients with breast cancer. Neoplasma. 1993; 40:81-85.

5 Liljefors M, Nilsson B, Hjelm Skog AL, et al. Natural killer (NK) cell function is a strong prognostic factor in colorectal carcinoma patients treated with the monoclonal antibody 17-1A. Int J Cancer. 2003; 105:717-723.

6 Pasero C, Gravis G, Granjeaud S, et al. Highly effective NK cells are associated with good prognosis in patients with metastatic prostate cancer. Oncotarget. 2015; 6:14360-14373.

7 Pirbudak Cocelli L, Ugur MG, Karadasli H. Comparison of effects of low-flow sevoflurane and desflurane anesthesia on neutrophil and T-cell populations. Curr Ther Res Clin Exp. 2012; 73:41-51.

8 Melamed R, Bar-Yosef S, Shakhar G, Shakhar K, Ben-Eliyahu S. Suppression of natural killer cell activity and promotion of tumor metastasis by ketamine, thiopental, and halothane, but not by propofol: mediating mechanisms and prophylactic measures. Anesth Analg. 2003; 97:1331-1339.

9 Shavit Y, Ben-Eliyahu S, Zeidel A, Beilin B. Effects of fentanyl on natural killer cell activity and on resistance to tumor metastasis in rats. Dose and timing study. Neuroimmunomodulation. 2004; 11:255-260.

10 Colacchio TA, Yeager MP, Hildebrandt LW. Perioperative immunomodulation in cancer surgery. Am J Surg. 1994; 167:174-179.

11 Cronin AJ, Aucutt-Walter NM, Budinetz T, et al. Low-dose remifentanil infusion does not impair natural killer cell function in healthy volunteers. Br J Anaesth. 2003; 91:805-809.

12 Kwak JH, Baek SH, Woo Y, et al. Beneficial immunostimulatory effect of short-term Chlorella supplementation: enhancement of natural killer cell activity and early inflammatory response (randomized, double-blinded, placebo-controlled trial). Nutr J. 2012; 11:53.

13 Sacerdote P, Manfredi B, Mantegazza P, Panerai AE. Antinociceptive and immunosuppressive effects of opiate drugs: a structure-related activity study. Br J Pharmacol. 1997; 121:834-840.

14 Kushida A, Inada T, Shingu K. Enhancement of antitumor immunity after propofol treatment in mice. Immunopharmacol Immunotoxicol. 2007; 29:477-486.

15 Inada T, Kubo K, Shingu K. Possible link between cyclooxygenase-inhibiting and antitumor properties of propofol. J Anesth. 2011; 25:569-575.

16 Markovic SN, Knight PR, Murasko DM. Inhibition of interferon stimulation of natural killer cell activity in mice anesthetized with halothane or isoflurane. Anesthesiology. 1993; 78:700-706

17 Leahy KM, Ornberg RL, Wang Y, et al. Cyclooxygenase-2 inhibition by celecoxib reduces proliferation and induces apoptosis in angiogenic endothelial cells in vivo. Cancer Res. 2002; 62:625-631.
$18 \mathrm{Wu} \mathrm{Z}$, Frascaroli $\mathrm{G}$, Bayer $\mathrm{C}$, Schmal $\mathrm{T}$, Mertens $\mathrm{T}$. Interleukin-2 from Adaptive T Cells Enhances Natural Killer Cell Activity against Human Cytomegalovirus-Infected Macrophages. J Virol. 2015; 89:6435-6441.

19 Bhat R, Rommelaere J. NK-cell-dependent killing of colon carcinoma cells is mediated by natural cytotoxicity receptors (NCRs) and stimulated by parvovirus infection of target cells. BMC Cancer. 2013; 13:367.

20 Hori Y, Ibuki T, Hosokawa T, Tanaka Y. The effects of neurosurgical stress on peripheral lymphocyte subpopulations. J Clin Anesth. 2003; 15:1-8.

21 Chen J, Deng Q, Pan Y, et al. Prognostic value of neutrophil-to-lymphocyte ratio in breast cancer. FEBS Open Bio. 2015; 5: 502-507.

22 Loop $\mathrm{T}$, Dovi-Akue $\mathrm{D}$, Frick $\mathrm{M}$, et al. Volatile anesthetics induce caspase-dependent, mitochondria-mediated apoptosis in human $\mathrm{T}$ lymphocytes in vitro. Anesthesiology. 2005; 102:1147-1157.

23 Pirttikangas CO, Perttila J, Salo M. Propofol emulsion reduces proliferative responses of lymphocytes from intensive care patients. Intensive Care Med. 1993; 19:299-302.

24 Jia L, Dong R, Zhang F, et al. Propofol Provides More Effective Protection for Circulating Lymphocytes Than Sevoflurane in Patients Undergoing Off-Pump Coronary Artery Bypass Graft Surgery. J Cardiothorac Vasc Anesth. 2015; 29:1172-1179.

25 Delogu G, Moretti S, Antonucci A, et al. Apoptogenic effect of fentanyl on freshly isolated peripheral blood lymphocytes. J Trauma. 2004; 57:75-81.

26 Narahara H, Kadoi Y, Hinohara H, Kunimoto F, Saito S. Comparative effects of flurbiprofen and fentanyl on natural killer cell cytotoxicity, lymphocyte subsets and cytokine concentrations in post-surgical intensive care unit patients: prospective, randomized study. J Anesth. 2013; 27:676-683.

27 Buckley A, McQuaid S, Johnson P, Buggy DJ. Effect of anaesthetic technique on the natural killer cell anti-tumour activity of serum from women undergoing breast cancer surgery: a pilot study. Br J Anaesth. 2014; 113 (Suppl 1):i56-62.

28 Bar-Yosef S, Melamed R, Page GG, et al. Attenuation of the tumor-promoting effect of surgery by spinal blockade in rats. Anesthesiology. 2001; 94:1066-1073.

29 Conrick-Martin I, Kell MR, Buggy DJ. Meta-analysis of the effect of central neuraxial regional anesthesia compared with general anesthesia on postoperative natural killer T lymphocyte function. J Clin Anesth. 2012; 24:3-7.

30 Duske H, Sputtek A, Binder T, et al. Assessment of physiologic natural killer cell cytotoxicity in vitro. Hum Immunol 2011; 72:1007.

31 Park KH, Park H, Kim M, et al. Evaluation of NK cell function by flowcytometric measurement and impedance based assay using real-time cell electronic sensing system. Biomed Res Int 2013; 2013:210726. 